Originalveröffentlichung in: Tasso Borbé (Hrsg.), Semiotics Unfolding: Proceedings of the Second Congress of the International Association for Semiotic Studies, Vienna, July 1979, Den Haag 1984, Bd. 3, S. 1389-1396.

\title{
DIE FARBE IN DER MALEREI \\ SYMBOLISCHER UND SEMI-SYMBOLISCHER BEDEUTUNGSMODUS
}

Felix Thürlemann

METHODOLOGISCHE VORBEMERKUNGEN : FÜR EINE SEMIOTISCHE ANALYSE DER FARBE

An Farbtheorien besteht bekanntlich kein Mangel; trotzdem kann sich derjenige, der sich im Hinblick auf die Bedeutungsanalyse mit der Malerei befasst, nicht einfach auf eine gegebene Farbtheorie abstützen. So erweist sich etwa das von Munsell (1919) entwickelte kolorimetrische Modell zwar geeignet, die Totalität der Farbmanifestationen - mit einer wichtigen noch darzustellenden Einschränkung - klassierend $z u$ beschreiben; es ist jedoch ungeeignet, die chromatischen Kontraste $z u$ bestimmen, die in einem gegebenen Bildtext eine bedeutungsgenerierende Funktion übernehmen.

Als Einwand gegen die Möglichkeit einer semiotischen Analyse der Farbe oder gar gegen eine Semiotik visueller Systeme uberhaupt wird häufig vorgebracht, das menschliche Auge sei fähig, bis zu 600'000 verschiedene Farbtöne zu unterscheiden (so schon Kries, 1882); es sei deshalb von vorneherein unmöglich, eine der Phonologie entsprechende Analyse der chromatischen Substanz nach einer beschränkten Anzahl von Kategorien vorzunehmen. Dieser Einwand ist nicht stichhaltig, weil er das Problem der Relevanzebenen ausser acht lässt. Die Tatsache, dass das menschliche ohr mindestens eine gleich grosse Anzahl (in Tonhöhe und Tonfarbe) verschiedener Laute unterscheiden kann, verhindert nicht die Existenz von Sprachsystemen, innerhalb derer nur die Wahrnehmung einiger weniger akustischer Kontraste für den Verständigungsprozess relevant ist.

Wir gehen von der Hypothese aus, dass ein künstliches semiotisches Farbsystem im Verhältnis zu der von einer natürlichen Semiotik zur verfügung gestellten Farbtaxinomie eine modellierende Funktion erfüllt, und zwar in einem doppelten sinnel. Die Modellierung beinhaltet (1) die Bildung eines für einen bestimmten Maler oder eine Schule charakteristischen Farbvokabulars oder 'Palette' (durch Reduktion oder Verfeinerung der gegebenen Taxinomie) und (2) verschiedene Modi der Interpretation (Semiose) der Farbvokabeln und -kategorien.

Eine jeweilige natürliche Semiotik ist jedoch nicht allein durch ihre Farbtaxinomie bestimmt, sondern auch durch ein Wertsystem, wonach alle sinnlich wahrnehmbaren Substanzen, und damit auch die Farben, nach dem konnotativen Lektüremodus mit einer primären Bedeutung affektiver Natur belegt werden. So erscheinen Farben entweder als 'kalt' oder 'warm', 'hart' oder 'weich', 'sanft' oder 'aggressiv' usw. Goethe (1810) hat diesen primären Lektüremodus in seiner Farbenlehre unter dem Titel 'sinnlich-sittliche Wirkung der Farben' als erster systematisch besprochen $^{2}$. Wie wir sehen werden, ist der konnotativ-wertende Lektüremodus der Farbsubstanz auch innerhalb der künstlichen systeme, etwa dem der 
Malerei, wirksam und dient häufig dazu, die willkürliche semantische Interpretation der Farbkategorien und -vokabeln sekundār zu motivieren.

Bevor wir zwei mögliche Interpretationsmodi der Farben näher darstellen können, müssen einige Bemerkungen zum Begriff der Farbe selbst vorausgeschickt werden. Einer Anregung von A. J. Greimas folgend betrachten wir den einzelnen, manifestierten Farbton als ein zusammengesetztes; dieser kann in Analogie zum Phonem als eine Figur konstruiert werden, die aus relevanten chromatischen Merkmalen zusammengesetzt ist ${ }^{3}$. Wir postulieren also, dass jedem Bildtext ein paradigmatisches System von chromatischen Kategorien zugrunde liegt. Die chromatischen Kategorien können provisorisch mit den Parametern des kolorimetrischen Modells, Tonalität ${ }^{4}$, Sättigung und Helligkeit, identifiziert werden; dabei ist jedoch zu beachten, dass der Begriff der Sättigung schlecht definiert ist, und es sich bei der Kategorie der Tonalität um eine Archikategorie handelt, die als eine Hierarchie von Subkategorien weiter analysiert werden muss (vgl. Conklin, 1973).

Es hat sich gezeigt, dass die von Munsell (1919) gewählten Parameter zwar für die Bestimmung lichterzeugter Farbtöne ausreichen, nicht jedoch für die Analyse der Farben in der Malerei. Eine kohärente Analyse des Farbphänomens ist nur möglich, wenn man unter dem Begriff der chromatischen Kategorien alle diejenigen zusammenfasst, deren Kontraste diskriminatorische Funktion haben, d.h. die es erlauben, die gegebene Bildfläche in eine geschlossene Menge von Figuren und Grundflächen (im Sinne der Gestaltpsychologie) aufzuteilen. Die Analyse der Malerei verlangt dabei, neben den genannten Kategorien der Tonalität, Sättigung und Helligkeit, ebenfalls die Berücksichtigung von Kategorien der Materialität oder Oberflächenbeschaffenheit (wie rauh vs glatt, matt vs reflektierend) und der homogenen Flächenrasterung (wie punktiert vs schraffiert usw ${ }^{5}$.

Vom logischen Standpunkt aus können grundsätzlich zwei Typen von chromatischen Kategorien unterschieden werden, kategorielle und graduelle. Zum ersten Typ gehört z.B. die Kategorie der Tonalität: ein Farbton ist entweder grün oder blau, gelblich braun ( $\mathrm{d} . \mathrm{h}$. braun mit Gelb) usw. Auch Materialitäskontraste unterliegen dem kategoriellen Modus. Die Parameter der Sättigung und Helligkeit hingegen sind von gradueller Natur; Farbtöne sind mehr oder weniger hell und gesättigt.

Bei der Bestimmung der Kategorien ist primär der Standpunkt der Wahrnehmung massgebend. So können die Farbtöne physikalisch-optisch nach dem Parameter der Tonalität stufenlos verăndert werden (durch Variation der Wellenlänge; vgl. das Farbprisma), auf perzeptueller Ebene jedoch ist hier die Artikulation kategoriell (das menschliche Auge ist gezwungen das optisch homogene Farbspektrum in Streifen aufzugliedern).

\section{DER SYMBOLISCHE BEDEUTUNGSMODUS}

Das Gebiet der Farbensymbolik ist relativ häufig und eingehend, jedoch nicht immer mit einem ausreichenden methodischen Instrumentarium untersucht worden. Wir möchten hier den Begriff des symbolischen Interpretationsmodus im Bezug auf die Malerei an einem Beispiel etwas genauer darstellen. Unter dem symbolischen Interpretationsmodus verstehen wir nach Hjelmslev einen Semiose-Modus, bei dem für eine Einheit der 
Ausdrucksebene eine Einheit der Inhaltsebene gesetzt ist. Da vor allem die chromatischen Merkmale der Archikategorie der Tonalität leicht hypostasiert werden können (ein komplexer Farbton wird durch den entsprechenden Tonalitätsbegriff bezeichnet), ist es fast ausschliesslich diese chromatische Kategorie, die dem symbolischen Interpretationsmodus unterworfen wird. Ueberhaupt kann die These aufgestellt werden, dass sich nur Merkmale von Farbkategorien des kategoriellen Typus einer symbolischen Interpretation anbieten. Die Beispiele, etwa aus dem Bereich der Liturgie, sind allbekannt: /grün/ : : Hoffnung, /weiss/ : : Unschuld $d^{6}$.

Innerhalb der westlichen Bildtradition spielt der symbolische Bedeutungsmodus in der spätantike und im frühen Mittelalter eine besonders wichtige Rolle. Am besten untersucht ist die Geschichte der beiden Machtfarben Purpur und Gold, deren Bedeutungswert im spätantiken kaiserlichen Zeremoniell entwickelt und dann in die religiöse welt des Mittelalters übertragen wurde. Es ist leicht ersichtlich, dass die Interpretation dieser Farbbegriffe über den Materialwert der Pigmente (Metallgold, Saft der Purpurschnecke), die zu ihrer Darstellung verwendet wurden, motiviert ist ${ }^{7}$.

Als Beispiel für den symbolischen Interpretationsmodus soll eine Buchmalerei aus dem Perikopenbuch Heinrichs II, dem frühen 1l. Jahrhundert angehörend, genauer betrachtet werden ${ }^{8}$. Es lässt sich an diesem Beispiel aufzeigen, dass die symbolische Interpretation nicht auf der Ebene der manifestierten Farbtöne, sondern auf der abstrakten Ebene der chromatischen Kategorien spielt.

Wir wollen bei der Betrachtung der gewählten Malerei nur auf den Symbolwert des Farbbegriffs eingehen. Nach Haeberlein (1939: 118, Anm. 9) diente Gelb im Mittelalter häufig als Ersatz für die Machtfarbe Gold und schliesst sich somit im wesentlichen an seine Bedeutung an. In unserem Beispiel werden jedoch die Farbvokabeln Gold und Gelb nebeneinander und bedeutungsmässig leicht differenziert behandelt. Gold erscheint formelhaft für den Schmuckrahmen und die Nimben, Gelb dagegen hat eine strenge Zuordnung zu einem bestimmten semantischen Bereich. Die Tonalität /gelb/ steht für das Klassem 'himmlisch', nach der Formel: /gelb/ $\overrightarrow{:}$ 'himmlisch'9.

Die Tonalität/gelb/ erscheint in drei, jeweils leicht verschieden getönten, zwei- und dreigliedrigen, sättigungsvariierten Farbtonskalen, entsprechend der Grundformel: weiss - gelb - (ocker). Weiss und ocker müssen dabei als extreme sättigungsvarianten des zentralen Gelbtones aufgefasst werden. Die Sättigungsvariation dient dem Zweck der räumlichen Modellierung. Die drei Variationen der Grundskala und ihr jeweiliger Anwendungsbereich sind folgende (die bräunlich-schwarzen Konturlinien nicht eingerechnet):

(a) "weiss" - "mittleres Gelb" - "heller Ocker"

Obergewand der vier Engel und Wolke, auf der Christus steht.

(b) "weiss" - "bräunliches Gelb" - "dunkler Ocker"

Flügelpaare der unteren Engel.

(c) "weiss" - "fahles Gelb"

Inkarnatsfarbe allex himmlischen Figuren (Christus und die vier Engel), im Gegensatz zu den irdischen Akteuren, die eine rötliche Hautfarbe aufweisen 10 . 
Das angeführte Beispiel zeigt, dass ein Verständnis des symbolischen Bedeutungsmodus in der Malerei eine Analyse der manifestierten Farbtöne als Figuren von chromatischen Merkmalen voraussetzt. So ist im vorliegenden Fall nur die Kategorie der Tonalität, /gelb/, symbolisch relevant, nicht jedoch die jeweiligen sätigungsvarianten des geiben Farbtons als "weiss" oder gebrochen durch verschieden starke Braun- und Graubeimischungen.

\section{DER SEMI-SYMBOLISCHE BEDEUTUNGSMODUS}

Während der symbolische Bedeutungsmodus der Farbe in eingehenden Untersuchungen dargestellt, ja sogar im allgemeinen Bewusstsein gegenwärtig ist, gilt für den semi-symbolischen Bedeutungsmodus das Gegenteil. A. J. Greimas hat die semi-symbolische semiotische struktur in Ergänzung von Hjelmslevs Sprachtheorie als eine besondere Form neben der symbolischen Bedeutungsstruktur postuliert (Greimas und courtés, 1979: 343, 'sémiotique B.5.d'). Im Gegensatz zur symbolischen Interpretation, bei der je eine Einheit der Ausdrucks- und Inhaltsebene einander zugeordnet werden, sind es im Fall der semi-symbolischen semiosis nicht mehr Einheiten, sondern Kategorien (vgl. die Definition bei Greimas und Courtés 1979: 34, 'catégorie 5'). Das Beispiel, das Greimas anführt, stammt aus der Gestensprache. Wenn wir, um zuzustimmen, eine vertikale Kopfbewegung machen, um Neinzusagen eine horizontale, so sind dabei die Ausdruckskategorie der Richtung (vertikal vs horizontal) und die Inhaltskategorie der existentiellen Prädikation (Affirmation vs Negation) miteinander in Beziehung gesetzt. Zur Darstellung einer solchen kategoriellen zuordnung kann die Homologationsformel verwendet werden: /vertikal/ : /horizontal/ : Affirmation : Negation.

Für die semiotische Analyse der Malerei scheint sich der Begriff des semi-symbolischen Bedeutungsmodus als besonders fruchtbar zu erweisen, obwohl er, zumindest mit formalen Mitteln, bisher noch kaum untersucht worden istll. Wir wollen nun diesen Bedeutungsmodus am Beispiel einer Farbanalyse genauer darstellen.

Das Analyseobjekt, das wir gewählt haben, ist die konventionelle Farbskala, die in der flämischen Malerei des 17 . Jhds regelmässig bei der Darstellung von Sommerlandschaften verwendung findet ${ }^{2}$. Das Phänomen wird in der kunstgeschichtlichen Literatur als 'formule des trois tons' bezeichnet. Yvonne Thiéry (1953: 7) schreibt dazu in ihrer Untersuchung zur flämischen Landschaft des 17. Jhds: 'les plans nombreux sont divisés en trois groupes colorés, le premier en brun rougeâtre ou verdâtre, le second en vert, le dernier en bleu ou en gris bleuté'. Thiéry unterstreicht den konventionellen charakter dieser Formel, deren Anwendung weitgehend unabhängig von der objektfarbe der dargestellten Gegenstände ist.

Die Charakterisierung des Farbgebrauchs einzig nach der Kategorie der Tonalität, wie sie Thiéry vornimmt, erweist sich bei genauerer Betrachtung als ungenügend. Wir ziehen es vor, für die Beschreibung der flämischen Landschaft des 17 . Jhds eine ideale, fundamentale Farbskala zu postulieren, welche die Farbtöne "warmes, dunkles Braun" und "weisslich-helles Blau" an den Polen und ein "mittleres Grün" im Zentrum aufweist. Die absolute Helligkeit ist dabei kontinuierlich graduiert und 
die drei Tonalitäten Braun, Grün, Blau, sind durch Mischung (Braungrün und Grünblau) miteinander verschleift. Diese kontinuierliche Farbskala dient zur Simulierung der Raumtiefe, die somit vom semiotischen Standpunkt als ihr Inhalt angesehen werden kann. Dem hellen Blau entspricht der fernste, dem dunklen Braun der naheste Punkt.

Untersucht man diese Farbskala mit dem methodischen Instrumentarium, das wir in der Einleitung dargestellt haben, so erscheint sie als eine syntagmatische Anordnung von chromatischen Figuren, denen zwei relevante Kategorien, die Archikategorie der Tonalitäten und die Helligkeit zu grunde liegen. Dabei fällt auf, dass die Tonalitäten dem kategoriellen, die Helligkeit jedoch dem graduellen Typus angehören. Dieser ambivalenten logischen Natur entspricht eine zweifache semisymbolische Interpretation der Skala. Die polare Artikulation nach der Helligkeit (/extrem dunkel/ vs /extrem hell/) entspricht der graduellen Inhaltskategorie der Distanz (nahe vs fern), die Abfolge der drei Tonalitäten /braun/, /grün/, /blau/ jedoch wird trotz der Verschleifung kategoriell wahrgenommen und entspricht einer Dreigliederung des simulierten Raumes nach Vorder-, Mittel- und Hintergrund.

Die polare Artikulation nach der Distanz ist zudem motiviert durch die konnotativ-wertende Wahrnehmung der komplexen Farbtöne innerhalb der Skala entsprechend der thermischen Synästhesie: der dunkelbraune Farbton des nahen Pols erscheint als extrem 'warm', der hellblaue Farbton des fernen Pols als extrem 'kalt'. Dass die thermische opposition in der Malerei allgemein als eine Distanz-opposition wahrgenommen wird, ist bekannt (siehe etwa kandinsky, 1912 : 87).

Das folgende Schema versucht das Resultat der Analyse zusammenzufassen ( - bezeichnet graduelle, \|kategorielle oppositionen)13:

Schema 1. Farbskala der flämischen Landschaft (i . Jhd)

\begin{tabular}{|c|c|c|}
\hline & Kategorien & Merkmale \\
\hline $\begin{array}{l}\text { denotative } \\
\text { Inhaltsebene } \\
\text { (topologische } \\
\text { Kategorien) }\end{array}$ & $\begin{array}{l}\text { Distanz } \\
\text { Ebenen }\end{array}$ & $\begin{array}{l}\text { nah } \\
\text { vordergrund || Mittelgrund } \| \text { Hintergrund }\end{array}$ \\
\hline $\begin{array}{l}\text { konnotative } \\
\text { Inhal tsebene }\end{array}$ & $\begin{array}{l}\text { thermische } \\
\text { Synästhesie }\end{array}$ & 'warm' \\
\hline $\begin{array}{l}\text { Ausdrucksebene } \\
\text { (chromatische } \\
\text { Kategorien) }\end{array}$ & $\begin{array}{l}\text { Tonalität } \\
\text { Helligkeit }\end{array}$ & \begin{tabular}{|lcc} 
braun & $\|$ grün & |l blau \\
dunkel & hell
\end{tabular} \\
\hline
\end{tabular}

Die Analyse der Farbskala der flämischen Landschaft des 17. Jhas exlaubt es, abschliessend einige Thesen zu formulieren, die unserer Ansicht nach für die Farbanalyse im allgemeinen vor. Wichtigkeit sind: (1) Der häufig vorgebrachte Einwand, wonach die kontinuierliche Manifestation der Farbsubstanz eine semiotische Analyse verhindere, ist nicht stichhaltig. Entweder wird die Farbsubstanz bei der Wahrnehmung kategoriell artikuliert oder aber sie setzt, und dies ist bei den graduellen Kategorien der Fall, eine binäre Artikulation nach zwei extre- 
men Polen voraus.

(2) Das was Farbe genannt wird, ist semiotisch betrachtet ein komplexes Phänomen. Jeder manifestierte Farbton muss als eine dem Phonem vergleichbare Figur von verschiedenen chromatischen Kategorien konstruiert werden.

(3) Neben dem bekannten symbolischen Interpretationsmodus ist die semisymbolische Semiosis für die Farbanalyse, ja für die Analyse der Malerei überhaupt, von entscheidender Bedeutung.

Romanisches Seminar

Universität

Zürich, Schweiz

\section{ANMERKUNGEN}

1. Zum Begriff der 'natürlichen Semiotik' siehe Greimas und Courtés (1979: 250).

2. Die affektive Farbbedeutung ist bisher vor allem von der Psychologie innerhalb der Wahrnehmungstheorie und der Synästhesieforschung untersucht worden. Der bekannte Lüscher-Test (Lüscher, 1971), ein Affektivitätstest, baut auf dieser semiotischen Eigenschaft der Farben auf. Es ist interessant zu sehen, dass sich Lüscher weitgehend auf Kandinsky (1912) stützt, der die bisher wohl beste Beschreibung der affektiven Farbbedeutungen geliefert hat.

vom semiotischen Standpunkt aus hat Louis Hjelmslev (1954) einen wichtigen Beitrag zum Studium dieser Bedeutungsebene geliefert, der jedoch unseres wissens in der semiotischen Forschung noch nicht wieder aufgenommen worden ist. Hjelmslev behandelt das besprochene Phänomen unter dem Begriff 'niveau d'appréciations collectives' und verwendet den Begriff der Konnotation nicht. (Unser erweiterter Konnotationsbegriff stützt sich auf Greimas und Courtés, 1979). Im sprachlichen Bereich befasst sich die Phonostilistik mit dem gleichen Problem. Es sollte deshalb versucht werden, eine von den verschiedenen Substanzen unabhängige Theorie der konnotativ-wertenden Lektüre aufzubauen.

3. Zu diesem Ansatz vgl. J.-M. Floch 'Quelques positions pour une sémiotique visuelle' (Floch et al., 1980). Zum Begriff der 'Figur' siehe Greimas und Courtés (1979: 148f, 'figure 2').

4. Wir gebrauchen den Begriff 'Tonalität' (für engl. chroma und hue) an Stelle von 'Farbton' und reservieren den letzteren Begriff für die Bezeichnung der manifestierten komplexen Farbeinheit.

5. Diese letzte Kategorie oder vielmehr Archikategorie, die homogene Flächenrasterung, kann als sekundäre chromatische Kategorie bezeichnet werden. Es ist bekannt, dass in heraldischen. Handbüchern Tonalitätskontraste aus Druckkostengründen durch solche sekundäre chromatische Kontraste (Unterschiede in der Schraffur) ersetzt werden können. Die beiden genannten Kategorien wurden am Wiener Kongress von Ingrid Lempp unter dem Begriff der Textur untersucht.

6. Wir verwenden folgende Konventionen der Schreibweise: ' $:$ ' ist das Zeichen für die semiotische zuoranung (zu lesen: 'steht für'). Auf die linke Seite setzen wir den Begriff der Ausdrucksebene, auf die 
rechte den Begriff der Inhaltsebene. Der Begriff der Ausdrucksebene steht zwischen Schrägstrichen, um anzuzeigen, dass es sich um einen konstruierten chromatischen Begriff und nicht um einen manifestierten Farbton handelt.

7. Zur spätantiken und frühmittelalterlichen Farbensymbolik siehe etwa Haeberlein (1939).

8. Das Blatt ist in guten Farbreproduktionen relativ leicht zugänglich, so bei Schöne (1954: Titelblatt) und bei Haeberlein (1939: Tafel 3). Beide Abbildungen sind offenbar nach dem gleichen Klischeesatz hergestellt. Es handelt sich um seite $131 \mathrm{v}$ des cod. 1 at. 4452 der bayerischen Staatsbibliothek in München, einem werk der Reichenauer Malschule, entstanden zwischen 1002 und 1014. (Beschreibung des Codex bei Leidinger; dort jedoch nur schwarz-weisse Abbildung). Die Szene stellt die Himmelfahrt Christi dar. Christus entschwebt auf einer wolke in den Himmel, wo er von zwei Engeln empfangen wird. Auf der Erde stehen die Apostel und Maria, die von zwei Engeln ('Männern in weissen Gewändern', nach Apg. 1,10) getröstet werden. Es ist $z$ u beachten, dass nur die himmlischen Figuren sowie Maria und Petrus Nimben tragen, letztere gleichsam in Vertretung für die beiden Apostelgruppen links und rechts.

9. Die verschiedenen logischen Typen der Zuordnung zwischen Ausdrucksund Inhaltsmerkmalen, die in der Literatur zur Farbsymbolik nicht spezifiziert werden, müssten in einer umfassenden Analyse präzisiert werden. Hier z.B. ist sie nicht eineindeutig. /Gelb/ bezeichnet zwar ausschliesslich den himmlischen Bereich, das Umgekehrte ist jedoch nicht der Fall (die Flügel der oberen Engel haben einen Purpurton, den man auch bei den Gewändern der Apostel feststellen kann; ebenso erscheint die Gewandfarbe Weiss indifferent bei allen Eiguren). Wir haben deshalb das zuordnungssymbol ' : :' in der Formel mit einem Pfeil präzisiert.

10. Die Farbangaben bei Leidinger sind z.T. ungenau. Der auffallende Kontrast der Inkarnatsfarben in dieser Malerei wird von ihm merkwürdigerweise nicht erwähnt.

11. Erste Ansätze, diesen Begriff fruchtbar zu machen, enthält Floch (1980); siehe darin vor allem die Einzelanalysen von J.-M. Floch (zu Boubat) und des Verfassers (zu Klee) sowie im Beitrag von A. J. Greimas den Abschnitt III.1. 'sémiotique semi-symbolique'.

12. Eine grosse Anzahl solcher Gemälde ist in Le siècle de Rubens abge-

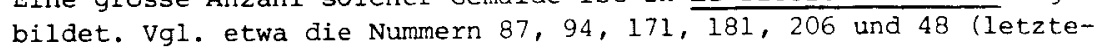
re mit farbiger Reproduktion).

13. Es ist $z u$ beachten, dass die beschriebene Farbskala eine abstrakte Grundformel darstellt. In Wirklichkeit geht mit ihr meistens eine sekundäre, gelb- oder graugetönte Farbskala parallel, die zur Darstellung beleuchteter Geländeabschnitte oder zur Modellierung von objekten dient. Die Simulation von Gegenständen kann jedoch auch allein über die Artikulation der ideal kontinuierlichen Farbskala geschehen. Figuren werden dort sichtbar, wo das Kontinuum der Skala durch Sprünge und vorausnahmen artikuliert wird. Dies entspricht unserem Postulat, wonach das Chromatische (Bereich der Farbe) und das Eidetische (Bereich der Form) komplementäre Dimensionen sind, und 
die Formwahrnehmung die Wahrnehmung chromatischer Kontraste voraussetzt.

\section{LITERATUR}

Conklin, Harold C. (1973). Color categorization (Rezension von B. Berlin und P. Kay Basic color terms). American Anthropologist 75: 931942.

Floch, Jean-Marie et al. (1980). De l'abstrait au figuratii. Paris. Goethe, Johann Wolfgang (1810). Farbenlehre, zit. nach Naturwissenschaftliche Schriften 1. Teil. Zürich/stuttgart: Artemis.

Greimas, Algirdas Julien und Courtés, Joseph (1979). Sémiotique: dictionnaire raisonné de la théorie du langage. Paris: Hachette.

Haeberlein, Fritz (1939). Grundzüge der nachantiken Farbikonographie. Römisches Jahrbuch für Kunstgeschichte 3: 75-126.

Hjelmslev, Louis (1954). La stratification du langage. Word 10: 163186; auch in: L. H. Essais 1inguistiques. Paris: Minuit 1971. Kandinsky, Wassily (1912). Ueber das Geistige in der Kunst. Zit. nach der 10. Aufl. Bern: Benteli 1973.

Kries, Johann von (1882). Die Gesichtsempfindungen und ihre Analyse. Leipzig.

Leidinger, Georg (o. d.). Miniaturen aus Handschriften der kgl. Hofund Staatsbibliothek in München. Heft 5: Das Perikopenbuch Kaiser Heinrichs des II. München.

Le siècle de Rubens (1978). Katalog der Ausstellung Paris, Grand-Palais, 17. Nov. $1977-13$. März 1978.

Lüscher, Max (1971). Der Lüscher Test: Persönlichkeitsbeurteilung durch Farbwahl. Hamburg: Rowohlt.

Munsell, Albert H. (1919). A color notation. Boston: Munsell Color Company.

Schöne, wolfgang (1954). Ueber das Licht in der Malerei. Berlin: Mann. Thiéry, Yvonne (1953). Le paysage flamand au XVIIe siècle. Paris/Bruxelles: Elsevier. 